\section{Métaux, pollution de l'air et santé}

\section{Les mousses, des alliées originales en épidémiologie}

\author{
Caroline Meyer ${ }^{1}$, Sébastien Leblond ${ }^{1}$, \\ Bénédicte Jacquemin ${ }^{2}$, દ́meline Lequy ${ }^{3}$
}

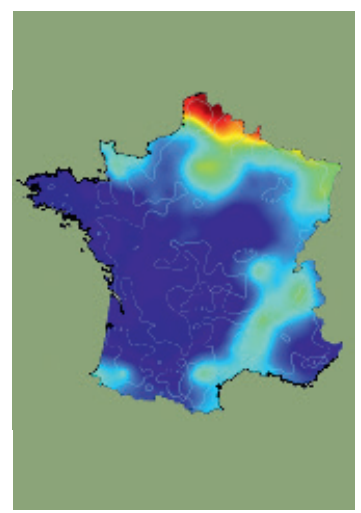

${ }^{1}$ UMS 2006 PatriNat (patrimoine naturel), Office français de la biodiversité (OFB), CNRS, Muséum national d'Histoire naturelle (MNHN), 12 rue Buffon, F-75005 Paris, France.

${ }^{2}$ Univ Rennes, Inserm, École des hautes études en santé publique (EHESP), Institut de recherche en santé, environnement et travail

(Irset) - UMR_S 1085,

9 avenue du Pr Léon Bernard, F-35000 Rennes, France.

des mécanismes de stress oxydant et d'inflammation pouvant expliquer certains effets sanitaires de la pollution de l'air [8]. Au-delà d'une ${ }^{3}$ Inserm UMS 011, 16 avenue Paul Vaillant Couturier, F-94807 Villejuif, France. emeline.lequy-flahault@inserm.fr certaine dose, les métaux deviennent en effet toxiques pour la santé et pour l'environnement [9]. Chez l'homme, cette toxicité a été révélée à la suite d'expositions accidentelles majeures à de fortes concentrations, notamment par ingestion, qui se sont traduites par l'apparition de symptômes particuliers: maladie de Minamata, causée par une exposition au mercure $(\mathrm{Hg})^{2}$, maladie d'Itai-Itai, par le cadmium $(\mathrm{Cd})^{3}$, ou saturnisme, par le plomb $(\mathrm{Pb})^{4}[10,11]$. En milieu professionnel, les vapeurs de certaines formes de $\mathrm{Cd}$ et de nickel $(\mathrm{Ni})$, qui peuvent être inhalées, sont classées cancérigènes [12]. La question des effets à long terme d'une exposition à de faibles concentrations de métaux, notamment par inhalation, reste beaucoup moins étudiée. Certains métaux atmosphériques font l'objet d'une réglementation et d'une surveillance de leur teneur ambiante par les autorités européennes, comme l'arsenic (As), le Cd, le $\mathrm{Ni}$, et le $\mathrm{Pb}$, dans les particules atmosphériques de moins de $10 \mu \mathrm{m}$

${ }^{2}$ De 1932 à 1966, une usine pétrochimique rejeta des métaux lourds, en particulier du mercure, dans la baie de Minamata, au Japon. Le mercure, ingéré par les poissons de la baie, s'est retrouvé dans les assiettes des habitants. Ceux-ci ont été sévèrement touchés par ce qui a ensuite été appelé « la maladie de Minamata » se traduisant par des symptômes neurologiques, sensoriels et moteurs.

${ }^{3}$ Cette maladie est apparue dès 1912 dans la ville de Toyoma, au Japon, en raison d'une intoxication au cadmium. Ce métal lourd a été rejeté en grande quantité par l'exploitation minière massive entre 1910 et 1945. La rivière locale, ainsi que ses affluents ont été très fortement pollués. Or cette rivière était utilisée pour irriguer les cultures de riz, mais aussi comme source d'eau de lavage et d'eau potable. Cette maladie provoque un ramollissement des os et une insuffisance rénale.

${ }^{4}$ Le saturnisme correspond à une imprégnation excessive des individus, en particulier des enfants, au plomb. Chez le jeune enfant, cela se traduit par une diminution des performances cognitives et sensorimotrices. Chez l'adulte, il augmente les risques de maladie rénale chronique et d'hypertension artérielle, altère la qualité du sperme et diminue la fertilité masculine. 
de diamètre $\left(P M_{10}\right)$. En France (métropolitaine et Outre-mer), cette surveillance est assurée par les Agences agréées de surveillance de la qualité de l'air (AASPA), dans un ensemble restreint de sites (50 pour le $\mathrm{Ni}, 56$ pour le $\mathrm{Pb}$ ). Ces sites de mesure demeurent peu nombreux en raison de difficultés techniques (conditions de non contamination des échantillons et seuils de détection des instruments de mesure actuels) et du coût d'exploitation. Ils permettent cependant de répondre aux exigences de la réglementation sur les métaux atmosphériques, sans toutefois apporter les données qui résulteraient d'un maillage fin et régulier du territoire.

Avec d'autres pays européens, la France participe au programme European Monitoring and Evaluation Programme qui fournit des modélisations des émissions et des dépôts de plusieurs métaux dans toute l'Europe. Les données obtenues avec ce programme ne permettent cependant pas d'obtenir une cartographie à fine résolution des expositions, en raison de la faible représentativité du terrain (mesures réalisées) ou du maillage trop large (modélisation). La biosurveillance par les mousses (des végétaux que l'on trouve dans tous les milieux terrestres) propose une alternative à ces évaluations atmosphériques afin d'estimer l'exposition des populations aux métaux sur de larges territoires.

\section{Les mousses bioaccumulatrices des métaux atmosphériques}

\section{Notions de biosurveillance}

La biosurveillance est définie comme «l'utilisation de systèmes biologiques (organismes et communautés d'organismes) pour suivre les changements de l'environnement dans l'espace et/ou dans le temps » [13]. La biosurveillance ne permet pas nécessairement d'identifier l'origine du polluant qui est mesuré, et certains organismes peuvent accumuler en grande quantité un ou plusieurs éléments provenant de leur environnement sans qu'il n'y ait d'impact majeur sur leur physiologie. On parle alors de bioaccumulateur. C'est le cas, par exemple, des mousses ou des lichens (résultant d'une symbiose entre un champignon et un organisme photosynthétique, cyanobactérie ou algue) qui accumulent les éléments atmosphériques, dont les métaux, à des niveaux supérieurs à ceux des limites de détection des instruments analytiques actuels.

\section{Les mousses : un organisme idéal pour la biosurveillance} de la qualité de l'air

Les mousses sont des organismes végétaux qui colonisent tous les milieux terrestres. Leurs propriétés morphologiques et physiologiques en font d'excellents capteurs des contaminants atmosphériques (Figure 1). En effet, l'absence de racine et de système vasculaire rendent ces végétaux dépendants de l'atmosphère pour leurs besoins nutritifs. L'absorption des nutriments est réalisée par toute la surface de la plante. Les mousses captent ainsi efficacement les éléments indispensables à leur croissance, mais capturent aussi simultanément un grand nombre d'éléments qui ne leurs sont pas essentiels, ce qui en fait des organismes de choix pour la biosurveillance de la qualité de l'air $[14,15]$.
Les métaux captés par le brin de mousse se localisent différemment selon leurs caractéristiques physicochimiques (Figure 1):

- les métaux particulaires se trouveront piégés à la surface du brin, entre les feuilles ;

- les métaux ioniques, en solution dans l'eau de pluie, rejoindront: l'espace extracellulaire, sur les sites de fixation de la paroi cellulaire; l'espace intercellulaire, sous forme d'ions libres; ou le milieu intra-cellulaire.

Généralement, seule la concentration totale en un élément est analysée, sans en différencier la forme chimique (particulaire ou ionique, état d'oxydation, etc.). Cette concentration permet d'estimer le niveau global d'exposition de la mousse de manière intégrée dans le temps. Cette technique est une méthode relative : elle permet de classer les sites de collecte les uns par rapport aux autres dans l'espace et le temps, en fonction de la valeur de concentration mesurée dans les échantillons. Elle n'a pas pour but de se substituer aux méthodes physico-chimiques, mais elle apporte des informations complémentaires. Elle permet de collecter des mesures par un maillage dense des territoires sur lesquels elle est déployée. Depuis le développement de cette technique dans les années 1960, les mousses ont été utilisées dans le cadre d'études à différentes échelles : au niveau local, au voisinage de sites industriels ou à l'échelle régionale, nationale et internationale.

\section{Un exemple d'utilisation aux niveaux européen et français : I'ICP-Vegetation et BRAMM}

Fondé sur des premières campagnes réalisées dans les années 1980 au Danemark et en Suède, le programme européen Atmospheric heavy metal deposition in Europe - estimation based on moss analysis a été par la suite étendu à d'autres pays d'Europe. À partir de 2001, ce programme a été intégré aux activités de la convention de Genève sur la pollution atmosphérique transfrontière, au travers de l'ICP-Vegetation (International cooperative programme on effects of air pollution on natural vegetation and crops). (e programme de coopération internationale étudie l'influence des métaux atmosphériques, ozone et azote, sur la végétation dans plus d'une trentaine de pays. L'un de ses objectifs est de mesurer les niveaux dits «de fond », c'est-à-dire loin des sources de contamination connues (comme les grands centres urbains ou les sites industriels), et de suivre les pollutions à longues distances selon un protocole défini [16]. Répété tous les cinq ans (à plus ou moins un an près en fonction des pays, en 1990-1991, 1995-1996, 2000-2001, 2005-2006, 2010-2011, 2015-2016, 2020), ce programme a inclus en 2010-2011 environ 4500 sites de prélèvement répartis sur 


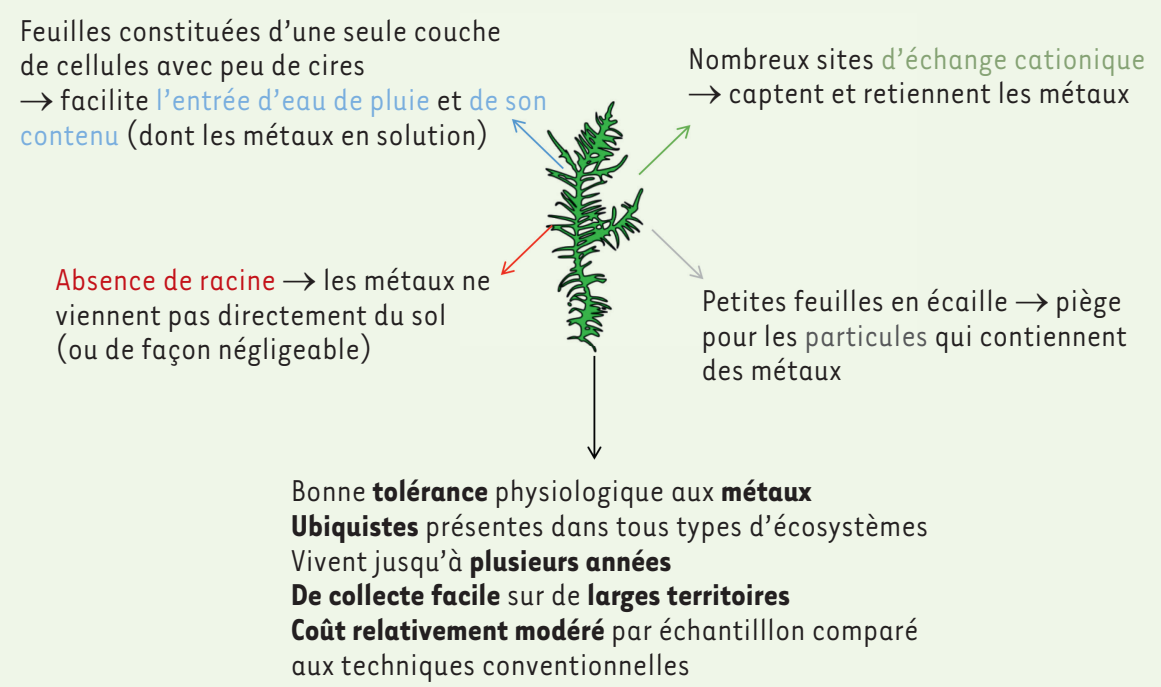

Figure 1. Caractéristiques permettant aux mousses d'être de bons bioaccumulateurs des métaux atmosphériques.

25 pays européens. Douze éléments métalliques - aluminium ( $\mathrm{Al}), \mathrm{As}, \mathrm{Cd}$, chrome ( $\mathrm{Cr}$ ), cuivre $(\mathrm{Cu})$, fer $(\mathrm{Fe}), \mathrm{Hg}, \mathrm{Ni}, \mathrm{Pb}$, antimoine $(\mathrm{Sb})$, vanadium $(V)$, zinc $(\mathrm{Zn})$ - sont analysés. Les données obtenues, fournies par les pays participants, ont ainsi permis de mettre en évidence une diminution au niveau européen des niveaux de $\mathrm{Pb}(77 \%)$ et de $\mathrm{Cd}(51 \%)$ entre 1990 et 2010 (concomitamment avec les baisses des émissions) [17].

La participation française à ce programme date de 1996, dans le cadre du dispositif BRAMM (Biosurveillance des retombées atmosphériques métalliques par les mousses) ${ }^{5}$, coordonné par le Muséum national d'histoire naturelle. Dans ce dispositif, les concentrations d'au moins 12 métaux sont évaluées sur plus de 450 sites répartis sur l'ensemble du territoire métropolitain. La dernière campagne de suivi a eu lieu en 2016. Elle a permis de mettre en évidence une diminution des niveaux de $\mathrm{Pb}$ entre 1996 et 2011 en France, mais aussi de révéler des variations temporelles moins évidentes pour les autres métaux (notamment le Cd) [18]. Le dispositif BRAMM permet aussi d'identifier des contrastes d'exposition au sein de la France métropolitaine, avec des régions plus ou moins contaminées, comme les bassins industriels et populationnels pour les métaux émis principalement par les activités humaines [19].

\section{Apport des mousses en épidémiologie}

L'idée d'utiliser des mousses ou des lichens comme « proxy » ${ }^{6}$ de l'exposition des populations aux métaux atmosphériques remonte aux années 1980 [20]. Comme les mousses, les lichens se nourrissent des éléments nutritifs apportés majoritairement par les dépôts atmosphériques et sont, eux aussi, utilisés comme témoins de la qualité de l'air. Selon les études, ils peuvent être utilisés comme accumulateur de polluants, ou

\footnotetext{
${ }^{5} \mathrm{http}: / /$ www.patrinat.fr/fr/biosurveillance-des-retombees-atmospheriques-metalliques-par-lesmousses-bramm-6071

${ }^{6}$ Un « proxy » permet d'évaluer une variable d'intérêt non observable ou non mesurable (ici, l'exposition humaine) en utilisant une variable qui la reflète (ici, les concentrations dans les mousses).
}

comme indicateur d'un niveau de pollution, en fonction de leur présence ou à cause de leur disparition.

Une étude réalisée en 1997 en Vénétie (Italie) [21], a ainsi révélé l'existence d'une corrélation entre la biodiversité des lichens présents sur les arbres, et la mortalité de la population par cancer. Cette étude pionnière est restée à un niveau écologique : elle comparait des données agrégées sur une même échelle géographique, sur un petit bassin de population. Dans la région du Neiss, entre Allemagne, République tchèque et Pologne, Wappelhorst et ses collègues ont de même établi des corrélations entre concentrations de métaux dans les mousses et incidence, entre 1993 et 1997, de maladies respiratoires (dans le cas du césium, du fer et du gallium) et maladies cardiovasculaires (pour le thallium) [22]. Aux Pays-Bas, Wolterbeek et Verburg ont analysé les corrélations entre les concentrations en métaux dosés dans les mousses et le taux de mortalité, entre 1993 et 1995 [23] : 30 métaux ont été examinés et, après analyse factorielle, neuf facteurs - assimilés à neuf sources de métaux - ont été corrélés à différentes causes de décès. Enfin, une étude réalisée au Portugal a exploré les associations entre les concentrations en métaux dans les lichens et la mortalité par cancer, en ajustant sur diverses covariables (à un niveau agrégé ${ }^{7}$ ) ; les résultats indiquaient des asso-

\footnotetext{
Les données agrégées ne sont pas collectées à un niveau individuel mais populationnel (et garantissent l'anonymat des individus interrogés) : par exemple, au niveau de la commune, du canton, de la région, ou dans le cas de l'étude portugaise, à un niveau subrégional défini par l'Union Européenne. Ces données sont le plus souvent sociodémographiques (pourcentage de femmes, de chômage, revenu moyen, etc) ou sanitaires (mortalité, incidence des maladies, etc.).
} 


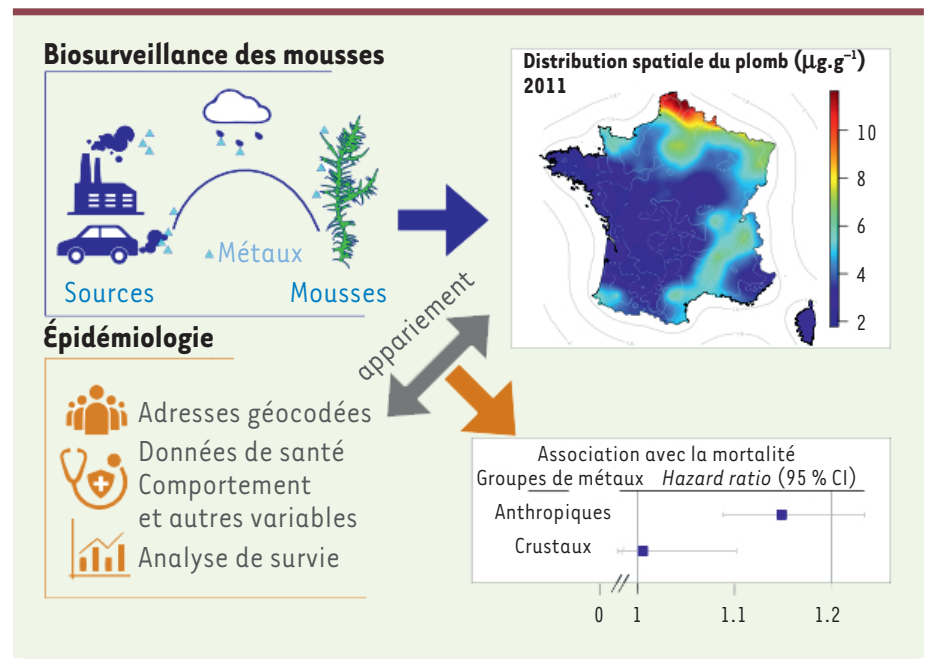

Figure 2. Principe général d'appariement des données de biosurveillance et d'épidémiologie pour analyser les relations entre exposition aux métaux de l'air et risque de mortalité naturelle en France. Les métaux sont émis par diverses sources et transportés dans l'atmosphère avant de se déposer sur les mousses. Les campagnes de biosurveillance incluent la collecte, la géolocalisation, et l'analyse des concentrations en métaux dans les mousses. Ces concentrations peuvent être interpolées sur toute la France par géostatistique (exemple du plomb en 2011). Ces estimations sont ensuite attribuées aux adresses des participants de la cohorte GAZEL, et groupées par analyse en composantes principales. L'analyse de survie permet d'estimer des associations entre exposition aux métaux et risque de mortalité, exprimé sous forme de «hazard ratio », ou rapport de risque entre les plus et les moins exposés.

ciations positives (mais pas forcément statistiquement significatives) entre $\mathrm{Ni}, \mathrm{Pb}$ et $\mathrm{V}$ et mortalité par cancer [24].

Ces travaux n'échappent pas aux limites des études écologiques utilisant des données agrégées, notamment, l'impossibilité d'ajuster finement sur des variables individuelles, et les difficultés d'interprétation des résultats au niveau populationnel et individuel. Elles présentent également certaines limites méthodologiques: une exposition peu précise avec des données non interpolées ou par simple pondération inverse, faute de structure spatiale ${ }^{8}$; des méthodes statistiques limitées à la corrélation ou à la régression linéaire multivariée, pour l'étude portugaise ; des résultats qui ne sont pas, parfois, concordants entre études (même en analyse univariée pour l'étude portugaise), ou avec la littérature, en particulier celle portant sur les métaux classés cancérigènes. Malgré ces limites, les résultats obtenus restent suffisamment intéressants pour motiver plus de recherches épidémiologiques utilisant les données de concentrations en métaux obtenues par bioaccumulation par les mousses ou les lichens.

\footnotetext{
${ }^{8}$ La structure spatiale comprend les variations spatiales de la mesure d'intérêt, ainsi que la quantification des amplitudes de variation, et les directions éventuellement différentes de ces variations. Si la mesure est aléatoire dans l'espace, elle est difficilement modélisable. Si elle n'est pas aléatoire, cette structure spatiale peut être modélisée en utilisant des outils statistiques adaptés aux données spatiales (géostatistiques), qui se basent entre autres sur la notion d'autocorrélation spatiale, c'est-à-dire la dépendance spatiale des valeurs prises par une variable en différents points de la zone d'étude.
}

En 2019 [25], une étude a été réalisée à partir des données épidémiologiques individuelles issues de la cohorte française GAZEL [26], constituée de 20000 volontaires ${ }^{9}$, et des données de concentration en métaux obtenues entre 1996 et 2011 par le dispositif BRAMM, à l'échelle de la France métropolitaine (sur environ 500 sites de collectes sur l'ensemble du territoire métropolitain). Ces données de concentration établies pour 13 métaux ont été interpolées grâce à une méthode géostatistique ${ }^{10}$, puis corrélées aux décès identifiés dans la cohorte.

Cette étude se voulait une «preuve de concept » quant à l'utilisation, dans une étude au niveau individuel, des données de biosurveillance issues des mousses en tant que marqueur d'exposition des populations (Figure 2). Les 13 métaux analysés au cours de 4 campagnes successives réalisées dans le contexte du dispositif BRAMM ont été regroupés selon deux méthodes : un regroupement supervisé ${ }^{11}$ fondé sur les données de la littérature, et un regroupement non-supervisé, déterminé par analyse en composantes principales ${ }^{12}$. Les deux méthodes distinguent les métaux d'origine majoritairement anthropiques (comme le $\mathrm{Pb}$ et le $\mathrm{Cd}$ ) des métaux majoritairement naturels (comme le calcium et le $\mathrm{Fe}$ ), et, pour l'analyse en composantes principales, d'un groupe de métaux d'origine marine (comme le $\mathrm{Na}$ ). L'étude se fonde sur une analyse de survie selon le modèle de Cox: le modèle utilise l'exposition aux métaux atmosphériques variant dans le temps, et est ajusté sur les principales covariables individuelles comportementales (tabac, alcool, etc.) et sociodémographiques (sexe, niveau d'études, etc.). Les résultats indiquent des associations positives entre exposition aux métaux d'origine majoritairement anthropique et mortalité due à une cause sans définition, une cause cardiovasculaire, ou une cause respiratoire.

Selon leurs formes d'émissions, les métaux atmosphériques qui sont accumulés par les mousses peuvent provenir majoritairement de particules, qui sont en elles-mêmes associées à une mortalité [4]. Pour valider I'hypothèse que les associations déterminées ont pour

\footnotetext{
${ }^{9}$ http://www.gazel.inserm.fr/fr/accueil

${ }^{10}$ Dans ce cas précis, le krigeage ordinaire : la structure spatiale est modélisée par un semi-variogramme, c'est-à-dire une fonction mathématique modélisant la variance entre toutes les paires de points d'une zone d'étude en fonction de la distance entre ces points. Cela permet de visualiser l'autocorrélation spatiale, et d'interpoler entre les points de manière plus fine qu'une interpolation linéaire, avec en outre une indication sur l'incertitude de la modélisation.

${ }^{11}$ L'analyste impose son choix de regroupement.

${ }^{12}$ Ce groupement non-supervisé est choisi par le programme d'analyse en fonction de critères imposés par l'analyste; dans le cas de l'analyse en composantes principales, l'objectif est de réduire le nombre de variables en «composantes principales », décorrélées entre elles et synthétisant l'information contenue dans des variables corrélées entre elles, le tout en expliquant le maximum possible de la variance de l'ensemble du jeu de données.
} 
origine les métaux constituant les particules et non les particules qui les contiennent, le modèle a été ajusté aux valeurs d'exposition aux particules fines, fournies par un modèle développé pour l'Europe [27]. L'association avec les métaux d'origine anthropique diminue mais persiste, ce qui met en évidence un effet des métaux indépendant de celui des particules.

Comme dans toutes les études observationnelles, la causalité est loin d'être établie: les métaux peuvent être les marqueurs de sources émettant d'autres polluants qui ne sont pas évalués à l'heure actuelle, et les mécanismes physiologiques sur lesquels reposent les relations observées restent mal définis. Cependant ces études ouvrent la voie à une recherche sur différents problèmes de santé et invitent à explorer les effets des différents contaminants atmosphériques, leur synergie, et leurs modes d'action.

\section{Conclusion}

La biosurveillance de la qualité de l'air par les mousses est un outil efficace, reconnu depuis des décennies en sciences environnementales. Elle peut permettre de pallier les limites des jeux de données concernant les métaux atmosphériques qui sont fournis par les méthodes physico-chimiques. Utiliser les données de biosurveillance par les mousses comme marqueur d'exposition des populations aux métaux atmosphériques, à un niveau agrégé ou individuel, fournit des résultats complémentaires aux études sur les particules fines ou sur la pollution de l'air en général. En termes épidémiologiques, de futures recherches sont nécessaires pour relier ces valeurs d'expositions aux particules ainsi que pour mieux comprendre les relations entre les mesures dans les mousses et les effets observés.

De nombreux pays européens possèdent des dispositifs de surveillance de la qualité de l'air fondés sur l'accumulation des métaux par les mousses. Ces données pourraient être utilisées pour des études épidémiologiques régionales, nationales, voire même internationales. $\diamond$

\section{SUMMARY}

Metals, air pollution, and health: mosses to the rescue of epidemiology

While evidence has accumulated about the links between long-term exposure to air pollution and health, little is known about the health effects of airborne metals. In France, the law makes it mandatory to monitor atmospheric concentrations and deposition of some airborne metals, through measurements or modelling. But the available data are either too scarce or irregular, making them difficult to use in largescale epidemiology: using mosses in the wild offers a welcome alternative. Mosses belong to the few vegetal organisms able to accumulate airborne metals. As such, they have been used for decades in air quality survey networks in Europe. They provide data to assess population exposure to airborne metals and may complement classical research programmes on air pollution epidemiology. As an example, we estimated associations between exposure to airborne metals of anthropogenic origin and increased mortality in France. $\diamond$

\section{REMERCIEMENTS}

Les auteurs bénéficient du soutien financier de l'ADEME, de la Fondation ARC et de l'ANR.

\section{LIENS D'INTÉRÊT}

Les auteurs déclarent n'avoir aucun lien d'intérêt concernant les données publiées dans cet article.

\section{RÉFÉRENCES}

1. Vallero DA. Fundamentals of air pollution. New York : Academic Press, 2014 : $1000 \mathrm{p}$.

2. Thurston GD, Kipen H, Annesi-Maesano I, et al. A joint ERS/ATS policy statement: what constitutes an adverse health effect of air pollution? An analytical framework. Eur Respir J 2017 ; 49.

3. WHO. 2018.Ambient (outdoor) air quality and health. https://www.who.int/ news-room/fact-sheets/detail/ambient-(outdoor)-air-quality-and-health (accessed: 07-Dec-2018).

4. Pascal M, Chanel P de C, Wagner V, et al. The mortality impacts of fine particles in France. Science Total Environment 2016 ; 571 : 416-25.

5. Beelen R, Hoek G, Raaschou-Nielsen 0, et al. Natural-cause mortality and long-term exposure to particle components: an analysis of 19 European cohorts within the multi-center ESCAPE project. Environ Health Perspect $2015 ; 123: 525-33$.

6. Raaschou-Nielsen 0, Beelen R, Wang M, et al. Particulate matter air pollution components and risk for lung cancer. Environment International $2016 ; 87: 66-73$.

7. Ostro B, Lipsett M, Reynolds $P$, et al. Long-term exposure to constituents of fine particulate air pollution and mortality: results from the California teachers study. Environ Health Perspect 2010 ; 118 : 363-9.

8. Kelly FJ. Oxidative stress: its role in air pollution and adverse health effects. Occupational Environmental Medicine 2003 ; 60 : 612-6.

9. Gall JE, Boyd RS, Rajakaruna N. Transfer of heavy metals through terrestrial food webs: a review. Environmental Monitoring Assessment 2015 ; 187 : 201.

10. Andujar P, Bensefa-Colas L, Descatha A. Acute and chronic cadmium poisoning. Rev Med Interne 2010 ; 31 : 107-15.

11. Bensefa-Colas L, Andujar P, Descatha A. Mercury poisoning. Rev Med Interne $2011 ; 32: 416-24$.

12. International Agency for Research on Cancer. IARC monographs on the evaluation of carcinogenic risks to humans. Lyon : IARC, 2016.

13. Markert B. Definitions and principles for bioindication and biomonitoring of trace metals in the environment. J Trace Elements Medicine Biology 2007 ; 21 (suppl 1): 77-82.

14. Tyler G. Bryophytes and heavy metals: a literature review. Botanical J Linnean Society $1990 ; 104: 231-53$.

15. Bates JW. Mineral nutrient acquisition and retention by bryophytes. J Bryology $1992 ; 17: 223-40$.

16. Harmens H. Monitoring of atmospheric deposition of heavy metals, nitrogen and pops in europe using bryophytes. Monitoring manual. International Cooperative Programme on Effects of Air Pollution on Natural Vegetation and Crops, 2010.

17. Harmens $H$, Norris DA, Sharps K, et al. Heavy metal and nitrogen concentrations in mosses are declining across Europe whilst some hotspots remain in 2010 . Environmental Pollution $2015 ; 200: 93-104$

18. Lequy $\varepsilon$, Dubos $N$, Witté $I$, et al. Assessing temporal trends of trace metal concentrations in mosses over France between 1996 and 2011: a flexible and robust method to account for heterogeneous sampling strategies. Environmental Pollution 2017; 220 (part B) : 828-36.

19. Lequy $\varepsilon$, Saby NPA, Ilyin I, et al. Spatial analysis of trace elements in a moss bio-monitoring data over France by accounting for source, protocol and environmental parameters. Science Total Environment 2017 ; 590-1 : 602-10.

20. Gailey FA, Lloyd OL. Atmospheric metal pollution monitored by spherical moss bags: a case study of Armadale. Environ Health Perspect $1986 ; 68$ : 187-96.

21. Cislaghi C, Nimis PL. Lichens, air pollution and lung cancer. Nature 1997 ; $387: 463-4$.

22. Wappelhorst 0 , Kühn I, Oehlmann J, et al. Deposition and disease: a moss monitoring project as an approach to ascertaining potential connections. Science Total Environment $2000 ; 249$ : 243-56. 


\section{RÉFÉRENCES}

23. Wolterbeek HT, Verburg TG. Atmospheric metal deposition in a moss data correlation study with mortality and disease in the Netherlands. Science Total Environment $2004 ; 319$ : 53-64.

24. Sarmento S, Wolterbeek HTh, Verburg TG, et al. Correlating element atmospheric deposition and cancer mortality in Portugal: data handling and preliminary results. Environmental Pollution $2008 ; 151: 341-51$.

25. Lequy $\varepsilon$, Siemiatycki J, Leblond $S$, et al. Long-term exposure to atmospheric metals assessed by mosses and mortality in France. Environment International 2019 ; 129 : 145-53.
26. Goldberg M, Leclerc A, Zins M. Cohort profile update: the GAZEL cohort study. Int J Epidemiol $2015 ; 44: 77-77$ g.

27. Hoogh K de, Chen J, Gulliver J, et al. Spatial PM2.5, N02, 03 and BC models for Western Europe - Evaluation of spatiotemporal stability. Environment International 2018; $120: 81-92$

\section{TIRÉS À PART}

$\varepsilon$. Lequy
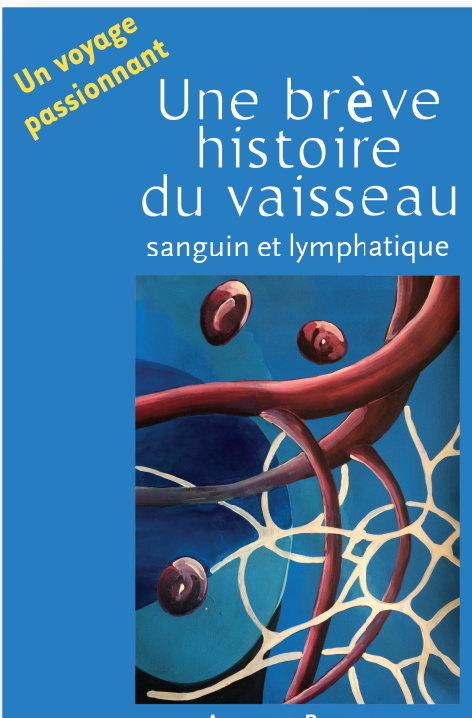

ANDREAS BIKFALVI livre, intéressant et lisible à la fois pour le spécialiste et le grand public, apporte des observations originales et nouvelles concernant l'angiogenèse, et notamment l'histoire des différentes découvertes, et discute les aspects et les concepts plus généraux en les plaçant dans le contexte de la philosophie des sciences.

Facile à lire, bien illustré, cet ouvrage cherche à comprendre et à faire comprendre les enjeux de la recherche sur l'arbre vasculaire en développement et en pathologie. II intéressera non seulement les étudiants et post-doctorants en biologie, mais aussi les chercheurs actifs dans ce domaine de recherche ainsi que toute personne intéressée par la biologie et la médecine et par l'histoire des sciences. Un voyage passionnant à travers l'histoire et les concepts les plus actuels concernant les recherches sur le vaisseau sanguin.

Andreas Bikfalvi est Professeur à l'université de Bordeaux et Directeur d'une unité de recherche Inserm sur le cancer et la biologie vasculaire. Il est, par ailleurs, membre senior de l'Institut Universitaire de France (IUF) et reconnu internationalement pour ses recherches dans le domaine de l'angiogenèse tumorale.

À retourner à EDP Sciences, 17, avenue du Hoggar, 91944 Les Ulis Cedex, France

Tél. : 0149856069 - Fax : 0149850345 - ع-mail : françois.flori@edpsciences.org

NOM :

Prénom :

Adresse :

Code postal :

Ville :

Pays :

Fonction :

Je souhaite recevoir

Une brève histoire de vaisseau : $25 €+3 €$ de port $=28 € \Pi C$

en ................. exemplaire, soit un total de

$\square$ Par chèque, à l'ordre de EDP Sciences

$\square$ Par carte bancaire : $\quad \square$ Visa $\square$ Eurocard/Mastercard

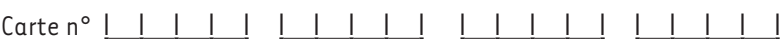

Date d'expiration: I I I I I I $N^{\circ}$ de contrôle au dos de la carte: I I I I I I Signature : 